\title{
Generalized Berreman's model of the elastic surface free energy of a nematic liquid crystal on a sawtoothed substrate
}

\author{
O. A. Rojas-Gómez and J. M. Romero-Enrique \\ Departamento de Física Atómica, Molecular y Nuclear, Area de Física Teórica Universidad de Sevilla, \\ Apartado de Correos 1065, 41080 Sevilla, Spain
}

(Received 18 May 2012; revised manuscript received 5 October 2012; published 26 October 2012)

\begin{abstract}
In this paper we present a generalization of Berreman's model for the elastic contribution to the surface free-energy density of a nematic liquid crystal in presence of a sawtooth substrate which favors homeotropic anchoring as a function of the wave number of the surface structure $q$, the tilt angle $\alpha$, and the surface anchoring strength $w$. In addition to the previously reported nonanalytic contribution proportional to $-q \ln q$, due to the nucleation of disclination lines at the wedge bottoms and apexes of the substrate, the next-to-leading contribution is proportional to $q$ for a given substrate roughness, in agreement with Berreman's predictions. We characterize this term, finding that it has two contributions: the deviations of the nematic director field with respect to a reference field corresponding to the isolated disclination lines and their associated core free energies. Comparison with the results obtained from the Landau-de Gennes model shows that our model is quite accurate in the limit $w L>1$, when strong anchoring conditions are effectively achieved.
\end{abstract}

DOI: 10.1103/PhysRevE.86.041706

PACS number(s): 61.30.Dk, 61.30.Hn, 61.30.Jf

\section{INTRODUCTION}

The behavior of nematic liquid crystals in the presence of microstructured substrates has been the subject of intensive research in recent times [1-3]. This problem has practical applications such as the design of zenithally bistable devices [4-9] or the trapping of colloidal particles in specified sites $[10,11]$. It is well known that the nematic director field, in the presence of a structured substrate, may be distorted, leading to an elastic contribution to the free energy. Since the seminal work by Berreman [12,13], this problem has been extensively studied and generalized in the literature [4,14-27]. Wetting and filling transitions by nematic on these grooved surfaces [28-31] have also been studied. When the substrate presents cusps, topological defects nucleate on them [14-16,26,27], and Berreman's expression of the elastic contribution to the freeenergy density, which is proportional to the wave number of the substrate structure $q$, breaks down because of the emergence of a nonanalytical contribution proportional to $-q \ln q$ associated to the nucleated defects [27]. This result contrasts with the phenomenology observed in smooth substrates, as sinusoidal substrates, in the absence of topological defects. In this situation a suitable generalization of Berreman's model may be applied [24,31].

In this paper we will complete the characterization of the elastic contribution to the surface free-energy density for sawtoothed substrates [27]. Beyond the $-q \ln q$ term previously mentioned, we find that the next-to-leading contribution follows Berreman's scaling with the wave number $q$. The origin of this term is twofold: (i) the deviations of the nematic director field with respect to the distortions imposed by the presence of the nucleated disclination lines and (ii) the defect core contributions. We estimate both contributions, finding a fairly good agreement with the reported values in Ref. [27].

The paper is organized as follows. The problem is set up in Sec. II, where we identify the different contributions to the elastic contribution to the surface free-energy density. Secs. III and IV are devoted to the estimation of these contributions, and the obtained results will be discussed in Sec. IV. The conclusions are presented in Sec. V.

\section{THE MODEL}

We consider a nematic liquid in contact with a sawtooth substrate characterized by the angle $\alpha$ and the length side $L$ (see Fig. 1). The substrate favors homeotropic anchoring of the molecules. We assume translational symmetry along the $z$ axis and a periodic distribution of wedges and cusps along the $x$ axis. Under these conditions, the nematic director field $\mathbf{n}(\mathbf{r})$ shows only in-plane distortions [27], so it can be parametrized by using the angle $\theta$ between the local director and the $y$ axis, yielding $\mathbf{n}(\mathbf{r})=(-\sin \theta(\mathbf{r}), \cos \theta(\mathbf{r}), 0)$. Far from the substrate, the bulk nematic phase orients homogeneously along either the $x$ axis (perpendicular texture $N^{\perp}$ ) or the $y$ axis (parallel texture $N^{\|}$). The nematic order of the system can be locally represented by a traceless symmetric orderparameter second-rank tensor $\mathbf{Q}$, with Cartesian components $Q_{i j}=\frac{3}{2} S\left[n_{i} n_{j}-\frac{1}{3} \delta_{i j}\right]+\frac{1}{2} B\left[l_{i} l_{j}-m_{i} m_{j}\right]$, where $S$ is the nematic order parameter, which measures the orientational ordering along the nematic director, and $B$ the biaxiality parameter, which measures the ordering of the molecules on the orientations perpendicular to $\mathbf{n}$, characterized by the eigenvectors $\mathbf{I}$ and $\mathbf{m}$.

As in previous works [27,29,30], the system will be described within the Landau-de Gennes (LdG) framework. The order-parameter tensor profile is obtained by minimizing the surface free-energy density functional

$$
\mathcal{F}_{\mathrm{LdG}}=\int_{\mathcal{V}}\left(\phi_{\text {bulk }}+\phi_{\mathrm{el}}\right) d V+\int_{\mathcal{A}} \phi_{\text {surf }} d s,
$$

where $\phi_{\text {bulk }}$ is the bulk free-energy density, $\phi_{\mathrm{el}}$ is the elastic free-energy density, and $\phi_{\text {surf }}$ is the surface free energy, defined as Ref. [13]

$$
\begin{gathered}
\phi_{\text {bulk }}=a \operatorname{Tr} \mathbf{Q}^{2}-b \operatorname{Tr} \mathbf{Q}^{3}+c\left[\operatorname{Tr} \mathbf{Q}^{2}\right]^{2}, \\
\phi_{\mathrm{el}}=\frac{\mathcal{L}_{1}}{2} \partial_{k} Q_{i j} \partial_{k} Q_{i j}+\frac{\mathcal{L}_{2}}{2} \partial_{j} Q_{i j} \partial_{k} Q_{i k}, \\
\phi_{\text {surf }}=-\frac{2}{3} \bar{w} \operatorname{Tr}\left[\mathbf{Q} \cdot \mathbf{Q}_{\text {surf }}\right],
\end{gathered}
$$




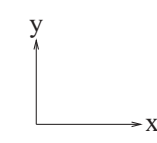

\section{Nematic}

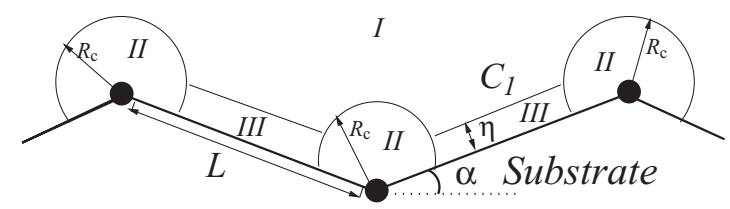

FIG. 1. Schematic picture of the geometry of the system, characterized by the side length $L$ and the angle $\alpha$. The different regions I, II, and III are outlined. See text for explanation.

where $a$ depends linearly on the temperature, $b$ and $c$ are positive constants, and $\mathcal{L}_{1}$ and $\mathcal{L}_{2}$ are positive parameters related to the elastic constants. This model shows a bulk nematic-isotropic transition for $a=b^{2} / 24 c$ (i.e., $\left.T=T_{\mathrm{NI}}\right)$, with a nematic order parameter $S_{b}(T)=b / 8 c+$ $(b / 8 c) \sqrt{1-64 a c / 3 b^{2}}$ below the transition. Note that the nematic order parameter in the nematic phase in coexistence with the isotropic phase is $S_{b}\left(T_{\mathrm{NI}}\right)=b / 6 c$. In the nematic phase, a natural length is given by the nematic correlation length $\xi$ along the local nematic director axis, which can be expressed in terms of the LdG model parameters as $\xi=\sqrt{\left(3 \mathcal{L}_{1} / 2+\mathcal{L}_{2}\right) /\left(9 b S_{b} / 4-6 a\right)}$. Finally, the free energy is reduced by $\xi S_{b}^{2}\left(3 \mathcal{L}_{1}+2 \mathcal{L}_{2}\right) / \sqrt{2}$. Regarding the surface contribution, homeotropic alignment of the nematic is favored by setting $\mathbf{Q}_{\text {surf }}=S_{\text {surf }}^{\text {ref }}(3 \boldsymbol{v} \otimes \boldsymbol{v}-1) / 2$, with $\boldsymbol{v}=$ $((x /|x|) \sin \alpha,-\cos \alpha, 0)$ being the outwards normal vector to the substrate and $S_{\text {surf }}^{\text {ref }}$ the favored nematic order parameter on the substrate. Note that Eq. (4) corresponds to the linearization on $\mathbf{Q}$ of the Nobili-Durand surface free energy [32]. This model favors a perfect nematic ordering on the surface (i.e., $S=1$ ), unlike the original Nobili-Durand surface contribution, which favors $S=S_{\text {surf }}^{\text {ref }}$. However, if $w$ and $S_{b}$ are small enough, the results obtained with both surface free energies are qualitatively similar. The nematic-substrate coupling Eq. (4) has been used to study the orientational wetting phenomena on flat $[33,34]$ and rough substrates [29-31,35].

As we are interested in surface phenomena, instead of minimizing directly the LdG functional Eq. (1), we subtract the contribution of an unperturbed bulk nematic phase to get the excess free energy and divide the result by the projection area of the substrate in the $x-z$ plane. In this way, and measuring all the quantities by their natural units described above, we get the following free-energy density functional:

$$
\begin{aligned}
\mathcal{F}= & \frac{1}{\lambda} \int_{-\lambda / 2}^{\lambda / 2} d x \int_{|x| \tan \alpha}^{\infty} d y\left[\frac{3-2 \tau}{4 \tau-3}\left(\frac{2 \operatorname{Tr} \tilde{\mathbf{Q}}^{2}}{3}-1\right)\right. \\
& -\frac{2}{4 \tau-3}\left(\frac{4}{3} \operatorname{Tr} \tilde{\mathbf{Q}}^{3}-1\right)+\frac{\tau}{4 \tau-3}\left(\frac{4}{9}\left[\operatorname{Tr} \tilde{\mathbf{Q}}^{2}\right]^{2}-1\right) \\
& \left.+\frac{1}{3+2 \kappa}\left[\partial_{k} \tilde{Q}_{i j} \partial_{k} \tilde{Q}_{i j}+\kappa \partial_{j} \tilde{Q}_{i j} \partial_{k} \tilde{Q}_{i k}\right]\right] \\
& -\left.\frac{1}{\lambda} \int_{-\lambda / 2}^{\lambda / 2} d x \frac{2}{3} w \operatorname{Tr}\left[\tilde{\mathbf{Q}} \cdot \tilde{\mathbf{Q}}_{\text {surf }}\right]\right|_{y=|x| \tan \alpha},
\end{aligned}
$$

where $\lambda \equiv 2 L \cos \alpha$ is the substrate periodicity wavelength, $\tilde{\mathbf{Q}}=\mathbf{Q} / S_{b}$, where $S_{b}=S_{b}(T)$ is the bulk value of the nematic order parameter at the temperature $T$ and $\tau=$ $S_{b}(T) / S_{b}\left(T_{\mathrm{NI}}\right)$ is the ratio between the nematic order parameter at the temperature $T$ and the nematic-isotropic transition temperature $T_{\mathrm{NI}}$. The positions and lengths are measured in units of $\sqrt{2} \xi$. Finally, $\kappa=\mathcal{L}_{2} / \mathcal{L}_{1}(\kappa>-3 / 2)$ and $w=\sqrt{8} \bar{w} \xi\left[S_{\text {surf }}^{\text {ref }} / S_{b}(T)\right] /\left(3 \mathcal{L}_{1}+2 \mathcal{L}_{2}\right)$ the reduced anchoring strength and $\tilde{\mathbf{Q}}_{\text {surf }}=(3 \boldsymbol{v} \otimes \boldsymbol{v}-1) / 2$. Hereafter, we consider every quantity in its corresponding natural units.

The global minimum of the functional Eq. (5) yields the mean-field equilibrium surface free-energy density, $f$, in reduced units. The contribution due to the elastic deformations induced by the substrate structure, $f_{\text {elastic }}$, can be obtained as $f_{\text {elastic }}=f-r \sigma_{\mathrm{NW}}(w)$, where the surface roughness is $r=$ $1 / \cos \alpha$ and $\sigma_{\mathrm{NW}}(w)$ is the nematic-flat substrate interfacial tension. In a similar way as it was obtained at nematic isotropic coexistence in Ref. [31], the interfacial tension for the LdG model has the expression

$$
\begin{aligned}
\sigma_{\mathrm{NW}}= & \frac{\sqrt{2} \sqrt{(\tau-1)(\tilde{S}(0)+1)^{2}+\tilde{S}(0)^{2}}}{6 \tau^{2} \sqrt{4 \tau-3}}\left[2 \tau^{2} \tilde{S}(0)^{2}-\tau(2 \tau+1) \tilde{S}(0)-4 \tau^{2}+7 \tau-3\right]-\frac{\sqrt{2} \sqrt{4 \tau-3}\left(-4 \tau^{2}+6 \tau-3\right)}{6 \tau^{2} \sqrt{4 \tau-3}} \\
& -\sqrt{2} \frac{1-3 \tau+2 \tau^{2}}{2 \tau^{5 / 2} \sqrt{4 \tau-3}} \ln \left[\frac{\tau \tilde{S}(0)+\tau-1+\sqrt{\tau} \sqrt{(\tau-1)(\tilde{S}(0)+1)^{2}+\tilde{S}(0)^{2}}}{2 \tau-1+\sqrt{\tau} \sqrt{4 \tau-3}}\right]-w \tilde{S}(0),
\end{aligned}
$$

where $\tilde{S}(0)=S(0) / S_{b}$ and $S(0)$ is the nematic order parameter at the substrate. The value of $\tilde{S}(0)$ is obtained as the largest solution of the equation

$$
(3-2 \tau) \tilde{S}^{2}(0)-2 \tilde{S}^{3}(0)+\tau \tilde{S}^{4}(0)=1-\tau+\frac{4 \tau-3}{2} w^{2} .
$$

At coexistence $\tau=1$ and Eq. (6) reduces to

$$
\sigma_{\mathrm{NW}}=\frac{\sqrt{2}(2 \tilde{S}(0)+1)(\tilde{S}(0)-1)^{2}}{6}-w \tilde{S}(0),
$$

where $\tilde{S}(0)=(1+\sqrt{1+2 \sqrt{2} w}) / 2$.
A systematic study of this system via full minimization of the LdG functional was done in Ref. [27]. For this purporse, the functional Eq. (5) was numerically minimized by using a conjugate-gradient method. The numerical discretization of the continuum problem is performed with a finite-element method [36] combined with adaptive meshing in order to resolve the different length scales that may emerge in the problem [37]. It was found that the $N^{\perp}$ texture has lower free energy if $\alpha<\pi / 4$ due to lesser distortion. Conversely, the $N^{\|}$texture has lower free energy for $\alpha>\pi / 4$, in agreement with earlier predictions [14-16,26]. For large $w L$, strong anchoring conditions are effectively achieved, leading to the 
nucleation of disclination lines characterized by non-halfinteger winding numbers along the ridges and wedges of the substrate [14-16,26,27]. As a consequence, the elastic contribution to the surface free-energy density has the following scaling [27]:

$$
f_{\text {elastic }} \approx-\frac{\mathcal{K}(\alpha)}{2 \pi} q \ln \frac{q \cos \alpha}{\pi}+\frac{q}{2 \pi} B(\alpha, w),
$$

where $q=2 \pi / \lambda=\pi / L \cos \alpha$ is the substrate periodicity wave number and $\mathcal{K}(\alpha)$ is defined as

$$
\mathcal{K}(\alpha)= \begin{cases}\frac{K \pi \alpha^{2}}{\left(\frac{\pi}{2}\right)^{2}-\alpha^{2}} & N^{\perp} \text { texture } \\ K \pi \frac{\pi}{\frac{\pi}{2}-\alpha} & N^{\|} \text {texture }\end{cases}
$$

with $K=(9 / 2)(2+\kappa) /(3+2 \kappa)$ being the reduced bulk elastic constant associated to bend and splay distortions. From the numerical results, the function $B$ is found to depend on the substrate roughness (i.e., $\alpha$ ) and nematic texture, as well as the anchoring $w$, but asymptotically not on $L$ for large $w L$. However, as $L$ increases, the complete minimization becomes very time-consuming. On the other hand, we do not get information about the origin of $B(\alpha, w)$.

A closer inspection of the typical nematic textures obtained from minimization of the $\mathrm{LdG}$ model for large $w L$ allows us to identify three different spacial regions with distinctive behavior in the nematic tensor field. Far from the substrates, the nematic order parameter $S$ takes the bulk value everywhere, but the orientational field $\theta$ deforms smoothly in order to satisfy the preferred anchoring conditions on the walls. Second, close to the walls but far away from apexes and wedges, there is a layer in which the orientation field is nearly homogeneous but $S$ changes in a scale of order of the nematic correlation length. In fact, the obtained nematic order parameter profile is basically equal to that obtained for a planar wall with the same anchoring conditions. Finally, around the apexes and wedges, both $S$ and $\theta$ are inhomogeneous. This explains the scaling of the surface free-energy density reported in Ref. [27] in terms of the substrate wavelength $\lambda$. Thus, the leading contribution to $f$ is $\sigma_{\mathrm{NW}}$ due to the fact that the anchoring is locally homeotropic on most of the substrate and that $S$ behaves as if the substrate were flat. On the other hand, the elastic deformations far away from the substrate can be described by the Frank-Oseen functional [27], which leads to a contribution of order of $\lambda^{-1}$ to $f$ in absence of topological defects and of order of $\ln \lambda / \lambda$ when disclination lines are present [29]. In both cases this contribution cannot overcome the leading order due to the surfaces if $L$ is large enough. Finally, we expect that the regions around the apexes and wedges will generate a line tension term, which contributes as $\lambda^{-1}$ to $f$. Inspired by these observations, in this paper we will introduce an alternative way to obtain $f_{\text {elastic }}$ from the functional Eq. (5). We divide the minimization domain into three regions (see Fig. 1): most of the domain will correspond to region I, formed by the points which are far enough from the substrate. The neighborhood of the substrate will be split into two regions: region II, formed by the union of the circular sections of radii $\xi<R_{c} \ll L$ centered at each wedge and apex, and region III, which are the points which are at a distance smaller than $\eta \sim \xi$ to the substrate, but at distances larger than $R_{c}$ from any substrate ridge. Our hypothesis is that, for large $w L$, the minimization of the surface free-energy functional restricted to each region (subject to appropriated boundary conditions) gives a good approximation to the complete minimization of $\mathcal{F}$. On the other hand, we anticipate that this analysis will give us some insight in the different contributions to $B(\alpha, w)$. However, the success of our approach can be assessed only when comparing their predictions with the results obtained from the full minimization of the LdG model, as will be discussed in Sec. V.

We start with the minimization of region III. As argued in Ref. [27], large $w L$ leads to strong anchoring conditions. This condition is equivalent to impose that $L$ is larger than the extrapolation length, which is of order of $K / w$ in reduced units. So, in order to minimize the surface free-energy density, we impose that the angle field $\theta(\mathbf{r})$ is constant along its boundary, so the nematic director field is homogeneous and equal to the normal to the substrate. Consequently, the minimization of the free-energy functional in this region will lead to a homogeneous director field normal to the substrate, although the nematic order parameter $S$ at each point will depend on its distance to the substrate. We impose the following fixed boundary conditions for $S: S=S_{b}$ at the boundary between regions I and III and the equilibrium nematic order parameter profile for the flat wall case at the boundary between regions II and III. Assuming that $\eta$ is large enough, this situation is completely equivalent to the flat case, so the minimum value of the surface free-energy density in this region, $f_{\mathrm{III}}$, will be $\sigma_{\mathrm{NW}}\left(1-2 R_{c} / L\right) / \cos \alpha$, with corrections of order of $\exp (-\eta / \xi)$. The next sections will be devoted to the evaluation of the minimum values of the surface free-energy functionals at the remaining regions, $f_{\mathrm{I}}$ and $f_{\mathrm{II}}$.

\section{EVALUATION OF $f_{\mathrm{I}}$}

The variations of $S$ are restricted to the neighborhood of the substrate of a width typically of order $\xi$ and inside the defect cores. So, in region I, $S$ takes the bulk value $S_{b}$ everywhere [27]. Thus, the surface free-energy functional to minimize in region I reduces to a Frank-Oseen functional,

$$
\mathcal{F}_{\mathrm{I}} \approx \frac{K}{2 \lambda} \int_{\mathrm{I}} d x d y|\nabla \theta|^{2}
$$

where the integration is restricted to region I, $K$ is the reduced elastic constant, and $\theta$ is the orientation field. The minimization of Eq. (11) yields to the Laplace equation for $\theta$, $\nabla^{2} \theta(\mathbf{r})=0$. In the far field, we impose Dirichlet boundary conditions $\lim _{y \rightarrow \infty} \theta(\mathbf{r})=\alpha_{\infty}$, where $\alpha_{\infty}=0$ for the $N^{\perp}$ texture and $\alpha_{\infty}=\pi / 2$ for the $N^{\|}$texture. Along the contours $x= \pm \lambda / 2$, we should impose periodic boundary conditions. However, we impose instead Dirichlet boundary conditions $\theta=\alpha_{\infty}$ along these contours, as we know from the full LdG model minimization that these are the conditions satisfied by the mean-field solution [27]. Finally, we assume strong anchoring conditions along the boundary between regions I and III: $\theta\left(x, y=x^{2} \tan \alpha /|x|, z\right)=\alpha_{\infty}+(x /|x|)\left(\alpha-\alpha_{\infty}\right)$. As discussed above, this condition will be accurate if $w L \gg 1$. Their contribution to the free-energy density, $f_{\mathrm{I}}$, comes from a contour integration of the mean-field solution via [27],

$$
f_{\mathrm{I}}=\frac{K\left(\alpha-\alpha_{\infty}\right)}{\lambda} \int_{C_{1}} \boldsymbol{v} \cdot \nabla \theta d \mathbf{s}
$$


where $C_{1}$ is the contour parallel to the boundary between regions I and III between a wedge and apex (see Fig. 1).

As argued in Refs. [14-16,27], the presence of cusps in the substrate induces the formation of disclination lines, which are responsible for the non-Berreman scaling of the elastic contribution to the surface free-energy density. In this section, we will complete that analysis, evaluating the next-to-leading contribution.

\section{A. Singular contribution}

The solution $\theta(\mathbf{r})$ to the Laplace equation subject to the boundary conditions mentioned above can be split into two terms: a singular contribution $\theta_{s}(\mathbf{r})$, due to the periodic array of disclination lines nucleated at the ridges of the substrate, and a nonsingular contribution, $\theta_{n s}(\mathbf{r})$. A representation of the singular contribution for each texture is given by Ref. [27]

$$
\begin{aligned}
\theta_{s}^{\perp}= & \frac{-\alpha}{\frac{\pi}{2}-\alpha}\left(-\arctan \left[\tan \frac{q x}{2} \operatorname{coth} \frac{q y}{2}\right]+\arctan \left[\tan \frac{q x}{2}\right]\right) \\
& +\frac{\alpha}{\frac{\pi}{2}+\alpha}\left(-\arctan \left[\tan \frac{q x}{2} \tanh \frac{q(y-L \sin \alpha)}{2}\right]+\arctan \left[\tan \frac{q x}{2}\right]\right) \\
\theta_{s}^{\|}= & \frac{\pi}{2}+\left(-\arctan \left[\tan \frac{q x}{2} \operatorname{coth} \frac{q y}{2}\right]+\arctan \left[\tan \frac{q x}{2}\right]\right) \\
& -\frac{\frac{\pi}{2}-\alpha}{\frac{\pi}{2}+\alpha}\left(-\arctan \left[\tan \frac{q x}{2} \tanh \frac{q(y-L \sin \alpha)}{2}\right]+\arctan \left[\tan \frac{q x}{2}\right]\right) .
\end{aligned}
$$

Their contribution to the free-energy density, $f_{\mathrm{I}}^{s}$, comes from a contour integration of these solutions,

$$
f_{\mathrm{I}}^{s}=\frac{K\left(\alpha-\alpha_{\infty}\right)}{\lambda} \int_{C_{1}} \boldsymbol{v} \cdot \nabla \theta_{s} d \mathbf{s}
$$

In Ref. [27] the large- $L$ behavior of Eq. (15) was estimated, leading to the non-Berreman term $\mathcal{K}(\alpha) q \ln \left(L / R_{c}\right)$. However, after some algebra, it is possible to obtain explicitly $f_{\mathrm{I}}^{s}$ from the solutions Eqs. (13) and (14) via Eq. (15) as

$f_{\mathrm{I}}^{s}=\frac{\mathcal{K}(\alpha) q}{2 \pi}\left\{-\ln q R_{c}+\ln \left[2 \cosh \left(\frac{\pi}{2} \tan \alpha\right)\right]-\alpha \tan \alpha\right\}$,

where $\mathcal{K}(\alpha)$ depends on the texture and substrate geometry as Eq. (10) and we neglected terms of order $q^{3}\left(R_{c}\right)^{2}$ and $q \eta$. As the dependence on the nematic texture comes from $\mathcal{K}(\alpha)$, $f_{\mathrm{I}}^{s}$ will be minimum for the $N^{\perp}$ (respectively, $N^{\|}$) texture for $\alpha<\pi / 4$ (respectively, $\alpha>\pi / 4$ ).

Two remarks are pertinent at this point. First, we note that although $f_{\mathrm{I}}^{s}$ may depend on the explicit representation of the singular solution, the leading non-Berreman contribution is independent on this representation. The reason for this is that this leading contribution arises from the behavior close to the wedges and apexes of $\theta_{s}$, which must asymptotically approach to the corresponding to an isolated disclination line [27]. Second, the next-to-leading contribution gives a first contribution to $B(\alpha, w)$, which we will denote as $B_{\mathrm{I}, s}(\alpha)$, given by the expression

$$
B_{\mathrm{I}, s}(\alpha)=\mathcal{K}(\alpha)\left(\ln \left[\frac{2}{\pi} \cosh \left(\frac{\pi}{2} \tan \alpha\right) \cos \alpha\right]-\alpha \tan \alpha\right) .
$$

\section{B. Nonsingular contribution}

The nonsingular part of the mean-field solution, $\theta_{\mathrm{ns}}$, is a solution of the Laplace equation $\nabla^{2} \theta_{\mathrm{ns}}=0$, subject to the boundary conditions $\theta_{\mathrm{ns}}=0$ in the far field, i.e., $y \rightarrow \infty$ and along the boundaries $x= \pm \lambda / 2$. On the other hand, $\theta_{\mathrm{ns}}=\alpha-\theta_{s}$ along $C_{1}$. We do not have an explicit expression for $\theta_{\mathrm{ns}}$ (however, there is an implicit expression via a SchwarzChristoffel transformation [14], see below), so we have to resort to numerical methods. We have used two different techniques: a finite-element method, analogous to the method outlined in Sec. II to solve the LdG model but minimizing, instead, the functional Eq. (11) subject to the boundary conditions for $\theta_{\mathrm{ns}}$ mentioned above, and, as an alternative, the boundary-element method [38,39]. In this technique the solution $\theta_{\mathrm{ns}}$ inside region I can be written as

$$
\begin{aligned}
\theta_{\mathrm{ns}}(\mathbf{r})= & \oint_{\partial I} d \mathbf{s}\left(\left[\mathbf{v}(\mathbf{s}) \cdot \nabla_{\mathbf{s}} \theta_{n s}(\mathbf{s})\right] G(\mathbf{s}, \mathbf{r})\right. \\
& \left.-\theta_{\mathrm{ns}}(\mathbf{s})\left[\boldsymbol{v}(\mathbf{s}) \cdot \nabla_{\mathbf{s}} G(\mathbf{s}, \mathbf{r})\right]\right),
\end{aligned}
$$

where the contour integral over the boundary $\partial I$ of region $I$ is counterclockwise, $\boldsymbol{v}(\mathbf{s})$ is the outwards normal to the boundary at $\mathbf{s}$, and $G(\mathbf{s}, \mathbf{r})$ is the fundamental solution of the Laplace equation $G(\mathbf{s}, \mathbf{r})=-\ln (|\mathbf{s}-\mathbf{r}|) / 2 \pi$. As we impose Dirichlet boundary conditions, the second term in the right-hand side of Eq. (18) is known. On the other hand, the normal derivative of $\theta_{\mathrm{ns}}$ at the boundary is obtained by solving the integral equation $[38,39]$

$$
\begin{aligned}
& \oint_{\partial I} d \mathbf{s}\left[\mathbf{v}(\mathbf{s}) \cdot \nabla_{\mathbf{s}} \theta_{\mathrm{ns}}(\mathbf{s})\right] G(\mathbf{s}, \mathbf{r}) \\
& =\frac{\theta_{\mathrm{ns}}(\mathbf{r})}{2}+\oint_{\partial I} d \mathbf{s} \theta_{\mathrm{ns}}(\mathbf{s})\left[v(\mathbf{s}) \cdot \nabla_{\mathbf{s}} G(\mathbf{s}, \mathbf{r})\right],
\end{aligned}
$$

where now $\mathbf{r} \in \partial I$. In order to solve Eq. (19), we discretize the boundary in a set of straight segments (the boundary elements). 


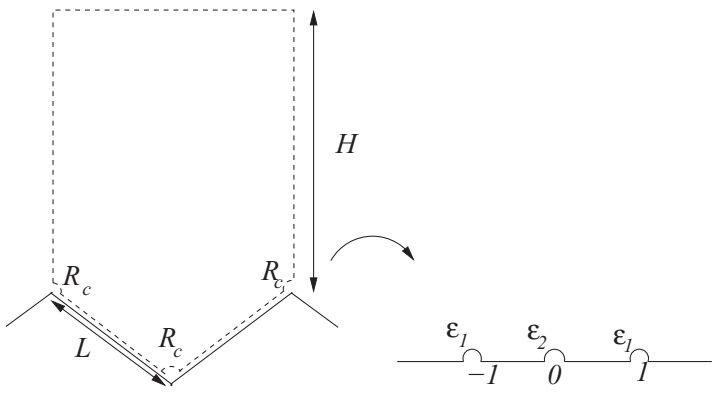

(a)

(b)

FIG. 2. (Left) Minimization cell for the evaluation of $f_{\mathrm{I}, \mathrm{ns}}$. (Right) Mapping of the minimization cell under the Schwarz-Christoffel transformation in the limit $H \rightarrow \infty$.

We use the constant boundary-element approach [39], so we assume that both $\theta_{\mathrm{ns}}$ and its normal derivative are constants along each boundary element. Introducing this approximation to Eq. (19), we obtain a set of linear algebraic equations for the normal derivatives of $\theta_{\mathrm{ns}}$. Once we solve this set of equations, and introducing the same approximation in Eq. (18), we obtain the nonsingular orientational field $\theta_{\mathrm{ns}}$ inside region I.

Once $\theta_{\mathrm{ns}}$ is evaluated, its contribution to the surface freeenergy density can be obtained from

$$
\begin{aligned}
f_{\mathrm{I}}^{\mathrm{ns}} & =\frac{K\left(\alpha-\alpha_{\infty}\right)}{\lambda} \int_{C_{1}} \boldsymbol{v} \cdot \nabla \theta_{\mathrm{ns}} d \mathbf{s} \\
& =\frac{K}{2 \lambda} \int_{\mathrm{I}} d \mathbf{r}\left|\nabla \theta_{\mathrm{ns}}\right|^{2}+\frac{K}{\lambda} \int_{C_{1}}\left[\alpha-\theta_{s}(\mathbf{s})\right] \boldsymbol{v} \cdot \nabla \theta_{s} d \mathbf{s},
\end{aligned}
$$

where the first result is more appropriated for the boundaryelement technique, while the second is more appropriate for the finite-element method [note that the last term in the second result can be evaluated numerically with high accuracy by standard methods as we know analytically $\left.\theta_{s}(\mathbf{r})\right]$.

The numerical minimization is performed in the cell shown in Fig. 2. In order to minimize finite-size effects, the cell height $H$ is taken to be at least 4 times the value of $L$ (note that $\theta_{s}$ decays exponentially to $\alpha_{\infty}$ for $\left.y \gg 2 L \cos \alpha / \pi\right)$. We checked that the value of $\lambda f_{\mathrm{I}}^{\text {ns }}$ is independent of $\lambda$, as expected, so the nonsingular contribution to $B(\alpha, w), B_{\mathrm{I}, \mathrm{ns}}(\alpha)$, is related to $f_{\mathrm{I}}^{\mathrm{ns}}$ via $B_{\mathrm{I}, \mathrm{ns}}(\alpha)=\lambda f_{\mathrm{I}}^{\text {ns }}$. The numerical results are shown for both the $N^{\perp}$ and $N^{\|}$textures in Fig. 3. The agreement between the results obtained from the finite-element method and boundary-element method is excellent, as for the evaluation of $\theta_{\mathrm{ns}}(\mathbf{r})$, which takes non-negligible values only above the contour $C_{1}$ and vanishes close to the wedges and apexes (see Fig. 4). Our results show that the nonsingular contribution corresponding to the $N^{\perp}$ (respectively, $N^{\|}$) texture is smaller than the contribution associated to the $N^{\|}$(respectively, $N^{\perp}$ ) texture for $\alpha<\pi / 4$ (respectively, $\alpha>\pi / 4$ ).

\section{Exact evaluation of $f_{\mathrm{I}}$}

In the previous paragraphs we have outlined how to obtain $\theta(\mathbf{r})=\theta_{s}(\mathbf{r})+\theta_{\mathrm{ns}}(\mathbf{r})$ and, from that, to obtain $f_{\mathrm{I}}=f_{\mathrm{I}}^{s}+f_{\mathrm{I}}^{\text {ns }}$, as well as $B_{\mathrm{I}}(\alpha)=B_{\mathrm{I}, s}(\alpha)+B_{\mathrm{I}, \mathrm{ns}}(\alpha)$. However, it is possible to obtain $f_{\mathrm{I}}$ directly without knowing the explicit form of $\theta(\mathbf{r})$

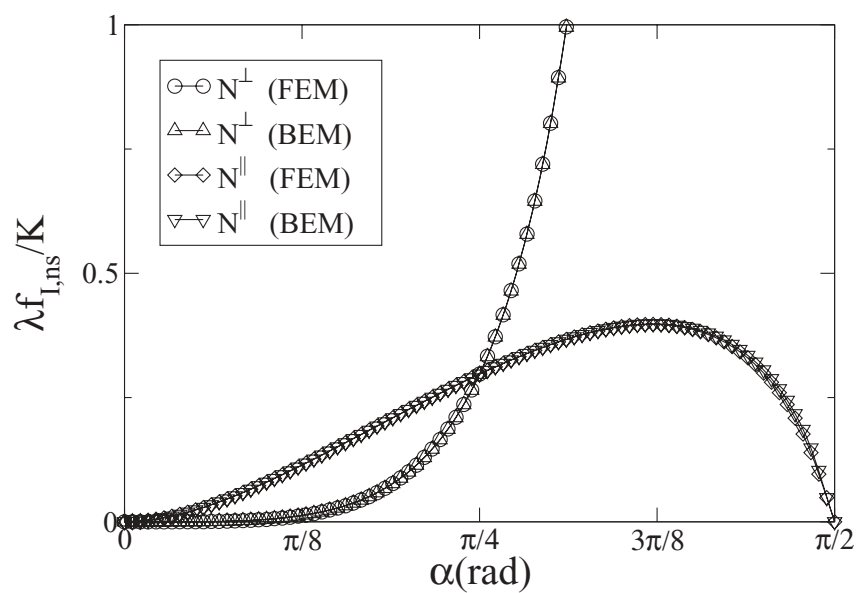

FIG. 3. Plot of $\lambda f_{\mathrm{I}, \mathrm{ns}} / K$ as a function of $\alpha$ for the $N^{\perp}$ and $N^{\|}$ textures by use of the finite-element method (FEM) and the boundaryelement method (BEM).

by using a Schwarz-Christoffel transformation [14,40],

$$
\begin{aligned}
z & =\int d \bar{\zeta} \frac{C}{(\tilde{\zeta}+1)^{1 / 2-\alpha / \pi} \tilde{\zeta}^{2 \alpha / \pi}(\tilde{\zeta}-1)^{1 / 2-\alpha / \pi}} \\
& =C^{\prime} \zeta^{1-\frac{2 \alpha}{\pi}}{ }_{2} F_{1}\left(\frac{1}{2}-\frac{\alpha}{\pi}, \frac{1}{2}-\frac{\alpha}{\pi}, \frac{3}{2}-\frac{\alpha}{\pi}, \zeta^{2}\right)+C^{\prime \prime}
\end{aligned}
$$

where $z=x+i y,{ }_{2} F_{1}(a, b, c, z)$ is the Gauss hypergeometric function, and $C, C^{\prime}$, and $C^{\prime \prime}$ are complex constants. This conformal transformation maps the minimization cell for $H \rightarrow \infty$ into the upper half $\zeta$ plane (see Fig. 2), transforming the origin into the origin and the edges $z= \pm L \cos \alpha+i L \sin \alpha$ into $\zeta= \pm 1$, respectively. These conditions fix the values of $C^{\prime}$ and $C^{\prime \prime}$, so the Schwarz-Christoffel transformation finally reads

$$
\begin{aligned}
z= & \frac{L e^{i \alpha}}{\Gamma\left(\frac{3}{2}-\frac{\alpha}{\pi}\right) \Gamma\left(\frac{1}{2}+\frac{\alpha}{\pi}\right)} \zeta^{1-\frac{2 \alpha}{\pi}} \\
& \times{ }_{2} F_{1}\left(\frac{1}{2}-\frac{\alpha}{\pi}, \frac{1}{2}-\frac{\alpha}{\pi}, \frac{3}{2}-\frac{\alpha}{\pi}, \zeta^{2}\right),
\end{aligned}
$$

where $\Gamma(x)$ is the complete gamma function. Equation (23) can be formally inverted, so $\zeta=\zeta(z / L ; \alpha)=x^{\prime}(x / L, y / L ; \alpha)+$ $i y^{\prime}(x / L, y / L ; \alpha)$. We will consider the limit $\eta / L \rightarrow 0$ and $R_{c} / L$ small but finite. In this approach, the boundary of zone II

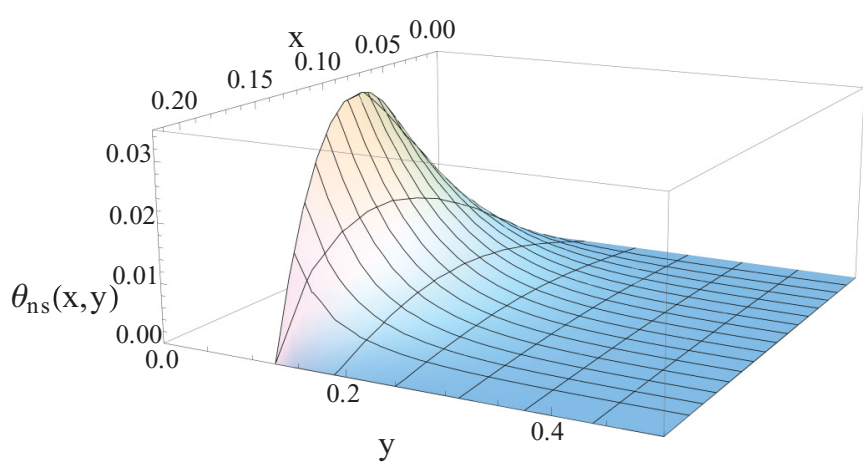

FIG. 4. (Color online) Plot of $\theta_{\mathrm{ns}}$ as a function of $x$ and $y$ obtained from the finite-element method for $\alpha=\pi / 6$ and $L=0.25$. For the sake of clarity it is only represented in half a minimization cell $0<$ $x<L \cos \alpha$. 
becomes, under the Schwarz-Christoffel transformation, the real axis in the $\zeta$ plane, rounded around $\zeta=0$ and \pm 1 . The expansion of Eq. (23) around these values show that the circles of radii $R_{c}$ around the origin and the edges $z= \pm L \cos \alpha+$ $i L \sin \alpha$ map into circles [up to corrections of order of $\left(R_{c} / L\right)^{2}$ ] of radii $\epsilon_{1}$ for $\zeta= \pm 1$ and $\epsilon_{2}$ for $\zeta=0$ given by

$$
\begin{aligned}
& \epsilon_{1}=\frac{1}{2}\left(\frac{1+\frac{2 \alpha}{\pi}}{1-\frac{2 \alpha}{\pi}}\right)^{\frac{1}{\frac{1}{2}+\frac{\alpha}{\pi}}}\left(\frac{R_{c}}{L} \Gamma\left[\frac{3}{2}-\frac{\alpha}{\pi}\right] \Gamma\left[\frac{1}{2}+\frac{\alpha}{\pi}\right]\right)^{\frac{1}{\frac{1}{2}+\frac{\alpha}{\pi}}} \\
& \epsilon_{2}=\left(\frac{R_{c}}{L} \Gamma\left[\frac{3}{2}-\frac{\alpha}{\pi}\right] \Gamma\left[\frac{1}{2}+\frac{\alpha}{\pi}\right]\right)^{\frac{1}{1-\frac{2 \alpha}{\pi}}} .
\end{aligned}
$$

As the Schwarz-Christoffel transformation is conformal, and $\theta(\mathbf{r})$ is harmonic, we are going to find the solution to the Laplace equation in the half $\zeta$ plane, $\tilde{\theta}$, subject to the boundary conditions $\tilde{\theta}=\alpha_{\infty}$ for $\left|x^{\prime}\right|>1+\epsilon_{1}$ and $\tilde{\theta}=$ $\alpha_{\infty}+\left(x^{\prime} /\left|x^{\prime}\right|\right)\left(\alpha-\alpha_{\infty}\right)$ for $\epsilon_{2}<\left|x^{\prime}\right|<1-\epsilon_{1}$. The solution $\tilde{\theta}\left(x^{\prime}, y^{\prime}\right)$ in the image of the region I on the $\zeta$ plane, $I^{\prime}$, is given by Ref. [14]

$$
\begin{aligned}
\tilde{\theta}\left(x^{\prime}, y^{\prime}\right)= & \alpha_{\infty}+\frac{\alpha-\alpha_{\infty}}{\pi} \arctan \frac{y^{\prime}}{x^{\prime}-1} \\
& -\frac{2\left(\alpha-\alpha_{\infty}\right)}{\pi} \arctan \frac{y^{\prime}}{x^{\prime}}+\frac{\alpha-\alpha_{\infty}}{\pi} \arctan \frac{y^{\prime}}{x^{\prime}+1} .
\end{aligned}
$$

From the solution $\tilde{\theta}$, we can obtain $\theta(x, y)=$ $\tilde{\theta}\left[x^{\prime}(x / L, y / L ; \alpha), y^{\prime}(x / L, y / L ; \alpha)\right]$. We note that we do not have an explicit expression for $x^{\prime}$ and $y^{\prime}$ as functions of $x$ and $y$, so we cannot give an analytic expression for $\theta(x, y)$. However, we can evaluate exactly $f_{\mathrm{I}}$ since

$$
\begin{aligned}
f_{\mathrm{I}} & =\frac{K}{2 \lambda} \int_{\mathrm{I}} d x d y|\nabla \theta|^{2}=\frac{K}{2 \lambda} \int_{I^{\prime}} d x^{\prime} d y^{\prime}\left|\nabla^{\prime} \tilde{\theta}\right|^{2} \\
& =\frac{K}{2 \lambda} \int_{\mathcal{B}} \tilde{\theta}\left(\boldsymbol{v}^{\prime} \cdot \nabla^{\prime} \tilde{\theta}\right) d \mathbf{s}^{\prime},
\end{aligned}
$$

where $\mathcal{B}$ is the image in the $\zeta$ plane of the boundary of zone $\mathrm{I}$. After a straightforward calculation, we get the expressions for $f_{\mathrm{I}}$ and $B_{\mathrm{I}}(\alpha)$,

$$
\begin{gathered}
f_{\mathrm{I}}=\frac{q \mathcal{K}(\alpha)}{2 \pi}\left[-\ln \frac{q R_{c} \cos \alpha}{\pi}-\ln \left(\Gamma\left[\frac{3}{2}-\frac{\alpha}{\pi}\right] \Gamma\left[\frac{1}{2}+\frac{\alpha}{\pi}\right]\right)\right. \\
\left.-\left(\frac{1}{2}-\frac{\alpha}{\pi}\right) \ln \left(\frac{\frac{\pi}{2}+\alpha}{\frac{\pi}{2}-\alpha}\right)\right] \\
B_{\mathrm{I}}(\alpha)=-\mathcal{K}(\alpha)\left[\left(\frac{1}{2}-\frac{\alpha}{\pi}\right) \ln \left(\frac{\frac{\pi}{2}+\alpha}{\frac{\pi}{2}-\alpha}\right)\right. \\
\left.+\ln \left(\Gamma\left[\frac{3}{2}-\frac{\alpha}{\pi}\right] \Gamma\left[\frac{1}{2}+\frac{\alpha}{\pi}\right]\right)\right]
\end{gathered}
$$

Figure 5 shows an excellent agreement between the theoretical prediction for $B_{\mathrm{I}}(\alpha)$ from Eq. (28) and the results obtained in the previous subsections, except close to $\alpha=\pi / 2$. The latter may be due to numerical uncertainties in the evaluation of $B_{\mathrm{I}, \mathrm{ns}}$, since either it diverges $\left(N^{\perp}\right.$ texture) or vanishes $\left(N^{\|}\right.$ texture) in that limit. As happened for $B_{\mathrm{I}, s}$, the dependence on the texture comes from $\mathcal{K}(\alpha)$, leading to the same conclusions about the relative stability of the nematic textures with the angle $\alpha$, in agreement with the results obtained in Ref. [14].

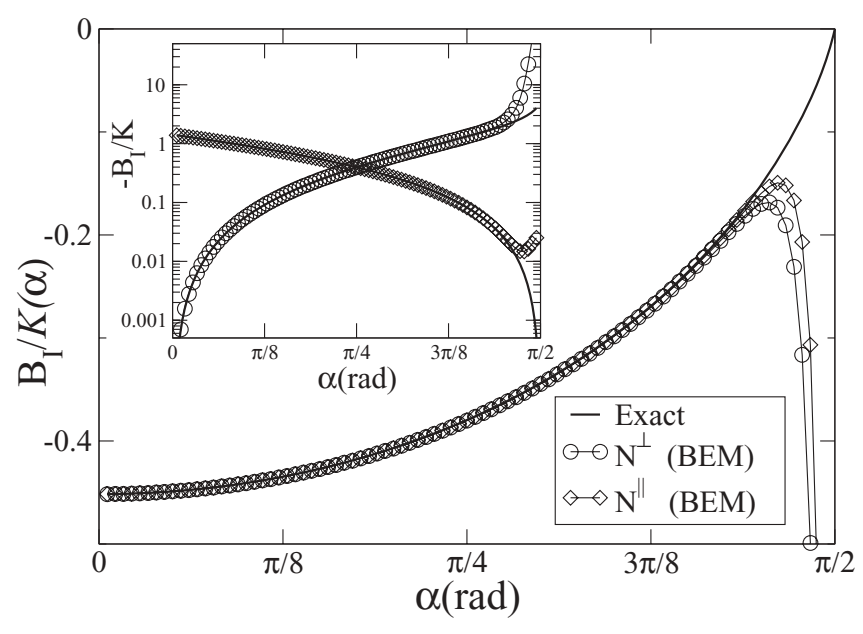

FIG. 5. Plot of $B_{\mathrm{I}} / \mathcal{K}(\alpha)$ as a function of $\alpha$. The continuous broad line corresponds to the exact expression, and the symbols correspond to the estimates from the numerical data for $f_{\mathrm{I}, \mathrm{ns}}$ (from the boundaryelement method): circles for the $N^{\perp}$ texture and diamonds for the $N^{\|}$ texture. (Inset) Plot of $-B_{\mathrm{I}} / K$ as a function of $\alpha$. The meaning of the symbols is the same as in the main panel.

\section{EVALUATION OF $f_{\text {II }}$}

In Ref. [27] it was observed that there is a dependence on $w$ of the next-to-leading contribution to the surface free-energy density. However, in the previous section we have shown that the contribution from region I depends only on $\alpha$. So we anticipate that this dependence comes from region II, where inhomogeneities of both the nematic order parameter $S$ and orientational $\theta$ fields are observed.

The free energy of region II can be evaluated as the sum of the contributions of the regions around each cusp (either wedge or apex). In each of these regions, and if $R_{c} \ll L$, we anticipate that the orientational field far from the cusp behaves asymptotically as that of the isolated disclination line which has been nucleated at the substrate wedge or apex: $\theta \sim I \phi$, where $I$ is the topological charge of the disclination line and $(r, \phi)$ are the polar coordinates taking as origin the cusp. The values of $I$ are fixed by the (strong) anchoring conditions on the substrate and depend on the nematic texture: $I_{1}=$ $-\alpha /(\pi / 2-\alpha)$ (respectively, $\left.I_{1}=+1\right)$ at the wedge bottom and $I_{2}=\alpha /(\pi / 2+\alpha)$ [respectively, $-(\pi / 2-\alpha) /(\pi / 2+\alpha)$ ] at the apexes for the $N^{\perp}$ (respectively, $N^{\|}$) [14,27]. However, $S$ decreases as $r \rightarrow 0$ from the bulk value far from the origin. Although we cannot solve analytically this problem, we can estimate, using an ansatz, the free energy associated to this combined distortion of $\theta$ and $S$ assuming a melted core (i.e., $S \rightarrow 0$ as $r \rightarrow 0$ ), as shown in the Appendix. However, we resort here to a full minimization of the $\mathrm{LdG}$ model [Eq. (5)] restricted to region II. At $r=R_{c}$, we impose Dirichlet boundary conditions to $\mathbf{Q}$, where $S$ takes the value corresponding to the order-parameter profile corresponding to a planar wall at the distance between the boundary point and the closest substrate, the biaxiality parameter $B=0$ and $\theta=\alpha_{\infty}+I(\phi-\pi / 2)$. Alternatively, we used free boundary conditions $\boldsymbol{v} \cdot \nabla \mathbf{Q}=0$, leading to similar results. Figure 6 shows typical textures obtained after minimization. These are similar to those obtained from full minimization close 


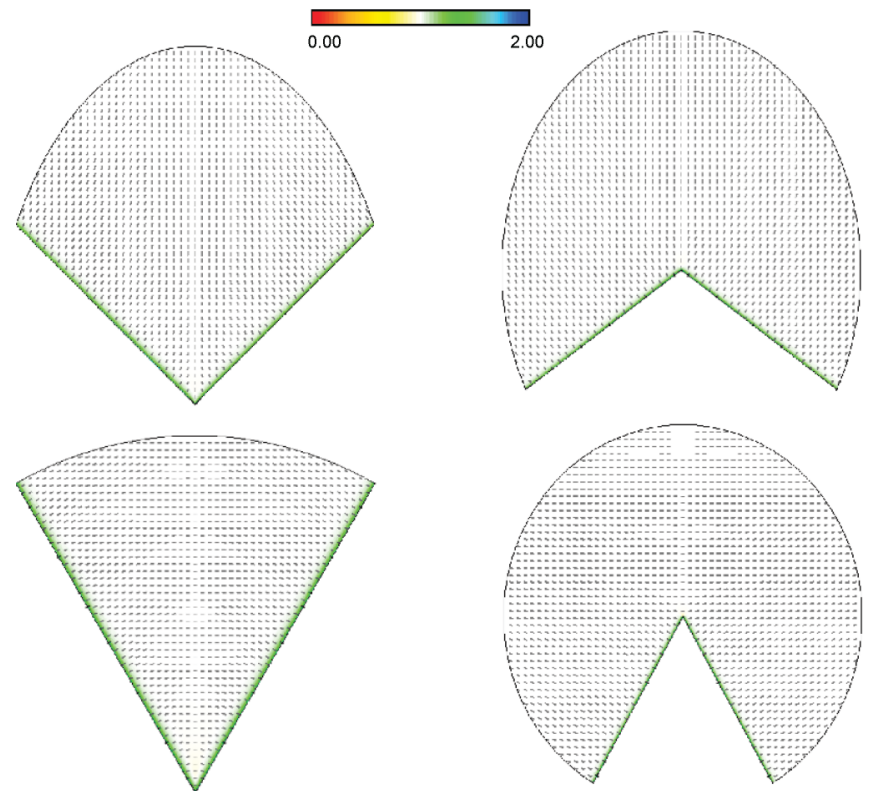

FIG. 6. (Color online) Typical textures obtained from the full minimization of the $\mathrm{LdG}$ function in region II for $\tau=1, \kappa=2$, $w=1.0, L=90$, and $\alpha=\pi / 6$ (upper panels) and $\alpha=\pi / 3$ (lower panels). The color map corresponds to the reduced nematic order parameter field $\tilde{S}=S / S_{b}$ and the segments correspond to the nematic director field $\mathbf{n}$.

to the cusps. At distances $r$ larger than a few correlation lengths, $S$ decays to the bulk value except in the neighborhood of the substrates, where it takes approximately the value corresponding to the flat substrate profile. In fact, the nematic order parameter profile in general shows only slight variations close to the cusps. This situation would correspond to a surface disorientation wall, in contrast with true disclination lines which exhibit melted cores. An exception to this general situation occurs when $\alpha \approx \pi / 4$, where a complex structure with a true defect line bound to the cusp is observed. On the other hand, the orientational field deforms continuously in order to satisfy anchoring conditions on each side of the wedge or the apex. So we anticipate that the contribution to the surface free-energy density $f_{\mathrm{II}}$ will scale with $R_{c}$ as

$$
\lambda f_{\mathrm{II}}=4 \sigma_{\mathrm{nw}} R_{c}+\mathcal{K}(\alpha) \ln R_{c}+B_{\mathrm{II}} .
$$

The first contribution arises from the inhomogeneities of $S$ close to the substrates and the second one from the asymptotic behavior of $\theta$ for $r>1$ : By using the Frank-Oseen functional Eq. (11), and taking into account that $\nabla \theta=I \mathbf{u}_{\phi} / r$ (where $\mathbf{u}_{\phi}$ is the azimuthal unit vector), we find that the free-energy contribution is proportional to $(K / 2) I^{2} \Delta \phi \ln R_{c}$, where $\Delta \phi$ is the opening angle of the wedge or apex. From this result, we get the second contribution in Eq. (29) by noting that $\mathcal{K}(\alpha)=K I_{1}^{2}(\pi-2 \alpha) / 2+K I_{2}^{2}(\pi+2 \alpha) / 2$. The remaining contribution will give the core free energy per unit length associated to the disclination lines.

Expression (29) can be used to extract $B_{\text {II }}$ from the numerical minimization. So fixing the value of $\alpha$, and for each $w$ (between 0 and 1.5), we considered a range of values of $R_{c}$ between 10 and 90 . The minimization was performed by
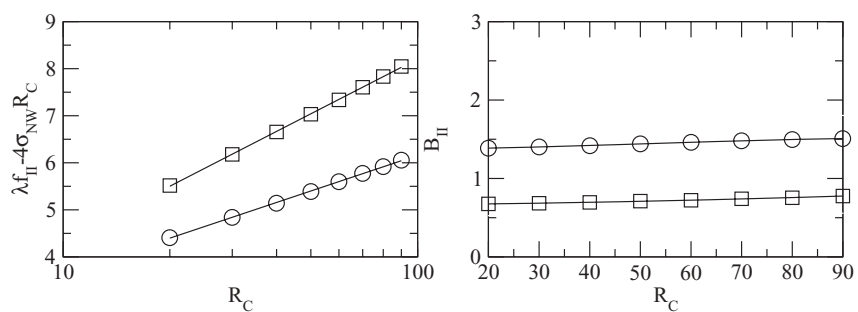

FIG. 7. (Left) Representation of $\lambda f_{\mathrm{II}}-4 \sigma_{\mathrm{NW}} R_{c}$ as a function of $R_{c}$ for $w=1$ and $\alpha=\pi / 6$ (squares) and $\alpha=\pi / 3$ (circles). The straight lines correspond to the logarithmic regressions of the numerical data. (Right) Representation of $B_{\mathrm{II}}(\alpha, w)$ as a function of $R_{c}$. The meaning of the symbols is the same as in the left panel.

the mesh-adaptive finite-element method used previously [37], taking $\tau=1$ and $\kappa=2$ (this choice is motivated to compare with results reported in the literature [27,29]). After substrating $4 \sigma_{\mathrm{nw}} R_{c}$ to $\lambda f_{\mathrm{II}}$, the numerical results clearly show a logarithmic dependence on $R_{c}$, with a slope approximately equal to $\mathcal{K}(\alpha)$ (see left panel in Fig. 7). The next step was to subtract the subdominant contribution $\mathcal{K}(\alpha) \ln R_{\infty}$. Now the numerical results are nearly independent of $R_{c}$ (see the right panel in Fig. 7). The value of $B_{\mathrm{II}}$ is estimated as the mean value of these results, with an error bar given by the dispersion of the numerical data around the average.

\section{DISCUSSION}

The results obtained in the previous sections can be combined as follows:

$$
f=f_{\mathrm{I}}+f_{\mathrm{II}}+f_{\mathrm{III}}=\frac{\sigma_{\mathrm{NW}}}{\cos \alpha}+f_{\text {elastic }},
$$

where

$$
\begin{aligned}
f_{\text {elastic }}= & \frac{q}{2 \pi} \mathcal{K}(\alpha)\left[-\ln \frac{q \cos \alpha}{\pi}-\left(\frac{1}{2}-\frac{\alpha}{\pi}\right) \ln \left(\frac{\frac{\pi}{2}+\alpha}{\frac{\pi}{2}-\alpha}\right)\right. \\
& \left.-\ln \left(\Gamma\left[\frac{3}{2}-\frac{\alpha}{\pi}\right] \Gamma\left[\frac{1}{2}+\frac{\alpha}{\pi}\right]\right)\right]+\frac{q}{2 \pi} B_{\mathrm{II}}(\alpha, w)
\end{aligned}
$$

up to corrections of order of $q^{2}$. Consequently,

$$
\begin{aligned}
B(\alpha, w)= & B_{\mathrm{I}}(\alpha)+B_{\mathrm{II}}(\alpha, w) \\
= & -\mathcal{K}(\alpha)\left[\left(\frac{1}{2}-\frac{\alpha}{\pi}\right) \ln \left(\frac{\frac{\pi}{2}+\alpha}{\frac{\pi}{2}-\alpha}\right)\right. \\
& \left.+\ln \left(\Gamma\left[\frac{3}{2}-\frac{\alpha}{\pi}\right] \Gamma\left[\frac{1}{2}+\frac{\alpha}{\pi}\right]\right)\right]+B_{\mathrm{II}}(\alpha, w) .
\end{aligned}
$$

This is the main result of our paper. We now can check this prediction by comparing these results with those obtained from the full minimization of the $\mathrm{LdG}$ model of a nematic in contact with a sawtoothed substrate [27]. In this reference, we considered NI coexistence conditions, $\kappa=2$, and a range of values of the fluid-substrate coupling $0.1<w<1.5$. Consequently, we are considering substrates with extrapolation lengths between $\xi$ and $10 \xi$. These values are smaller than the experimentally relevant situations even for strong anchoring 


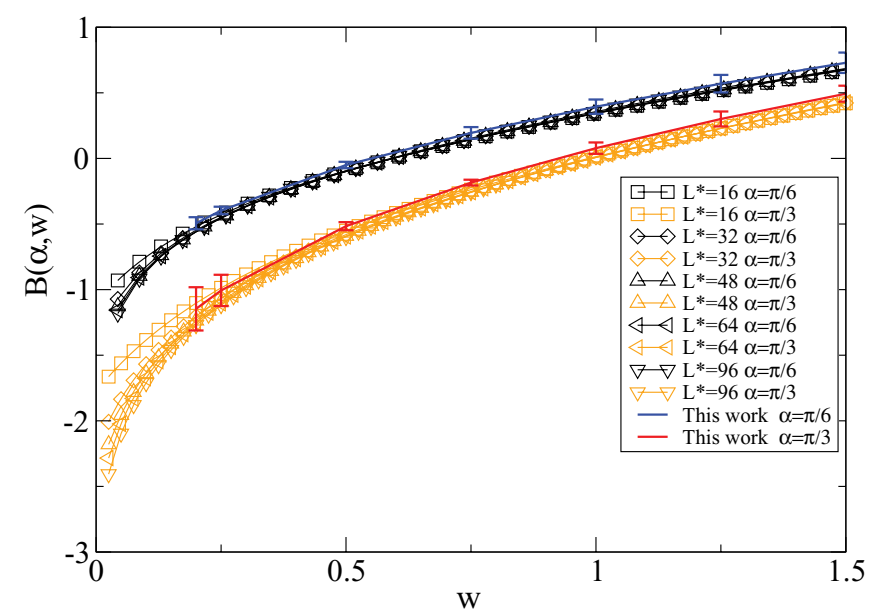

FIG. 8. (Color online) Comparison between the values of $B(\alpha, w)$ obtained from the full minimization of the LdG model [27] and the results obtained in the present work. Symbols correspond to $B(\alpha, w)$ obtained from the full minimization of the LdG model for different values of $L$ and $\alpha=\pi / 6$ in the $N^{\perp}$ texture (black symbols) and $\alpha=\pi / 3$ in the $N^{\|}$texture (orange or light gray symbols). The wide blue (black) and wide red (dark gray) lines with error bars correspond to the predictions from this work for $\alpha=\pi / 6$ and $\pi / 3$, respectively.

conditions (although under some circumstances they may be achieved [41]). On the other hand, the range of values of $L$, between 16 and 96, is also smaller than the experimentally feasible range. This range was dictated by computational limitations, as problems with the numerical procedure were observed for larger values of $L$. Our interest in the considered range of values of $w$ is twofold. First, this range covered the typical values of $w$ for which wetting and related phenomena on rough substrates were observed in our model [29-31,35]. Second, the collapse of the data for $B(\alpha, w)$ obtained for the considered values of $L$ was achieved only for $w>0.5$. However, we expect that this collapse will be observed also for smaller values of $w$ if we increase the range of $L$, and the trends shown in the available data support this claim.

Figure 8 shows the comparison between the results reported in Ref. [27] $(\tau=1, \kappa=2)$ and the calculated ones in this paper for $\alpha=\pi / 6\left(N^{\perp}\right.$ texture $)$ and $\alpha=\pi / 3\left(N^{\|}\right.$texture). For large $w$, the agreement is good, although our results slightly overestimate those from the full minimization. On the other hand, for small $w$ the curves obtained from the full minimization converge towards our prediction as $L$ increases. So, our approximation is accurate even for moderate values of $L$, despite the assumptions involved in our approach. Consequently, the scheme considered in this paper is an alternative to the full minimization technique, which is quite expensive from a computational point of view, when $w L>1$.

Finally, it is interesting to note that the elastic contribution to the surface free-energy density, Eq. (31), can be expressed as follows:

$$
\lambda f_{\text {elastic }}=2\left[2 \pi K \frac{I_{1}}{2} \frac{I_{2}}{2} \ln \frac{\gamma(\alpha)}{L}\right]+B_{\mathrm{II}}(w, \alpha),
$$

where we note that $\mathcal{K}(\alpha)=-4 \pi K\left(I_{1} / 2\right)\left(I_{2} / 2\right)$, with $I_{1}$ and $I_{2}$ being the topological charges associated to the disclination lines at the wedges and apexes, respectively. The characteristic length $\gamma(\alpha)$, which absorbs the contribution to $B$ from region I, is defined as

$$
\gamma(\alpha)=\Gamma\left[\frac{3}{2}-\frac{\alpha}{\pi}\right] \Gamma\left[\frac{1}{2}+\frac{\alpha}{\pi}\right]\left(\frac{\frac{\pi}{2}+\alpha}{\frac{\pi}{2}-\alpha}\right)^{\frac{1}{2}-\frac{\alpha}{\pi}} .
$$

This length scale decays continuously from $\pi / 2$ for $\alpha \rightarrow 0$ to 1 for $\alpha \rightarrow \pi / 2$. We can understand the first contribution in Eq. (33) as the interaction between the disclination line at $x=0$ with half its topological charge and the apexes at $x=$ $\pm L \cos \alpha$, again with half their topological charges [42]. As the absolute values of the topological charges are always smaller or equal to 1 , the nucleation of disclinations at the cusps of the surface is favorable with respect to bulk disclinations (which only may have half-integer values). With this intepretation, $\gamma(\alpha)$ can be used to define an effective core radius $\gamma(\alpha)$, and the sum of the core energies is given by $B_{\mathrm{II}}(\alpha, w)$.

\section{CONCLUSIONS}

In this paper we have analyzed the elastic contribution to the surface free-energy density for a nematic in contact to sawtoothed substrates in the strong anchoring regime, i.e., $w L>1$. We have extended the analysis done in Ref. [27]. So, in addition to the leading contribution proportional to $-q \ln q$, with $q$ being the substrate periodicity wave number $q$, we have characterized the next-to-leading term. This term has two contributions: one associated to the deviation of the orientational field with respect to the contribution of the array of disclination lines nucleated at the cusps of the substrate and the core free-energy associated to them. We anticipate that our analysis can be generalized for other substrate shapes when the nematic texture presents topological defects induced by the structure, as, for example, in crenellated substrates [35]. Furthermore, our results may be used to predict accurately the location of first-order wetting transitions in nematic liquid

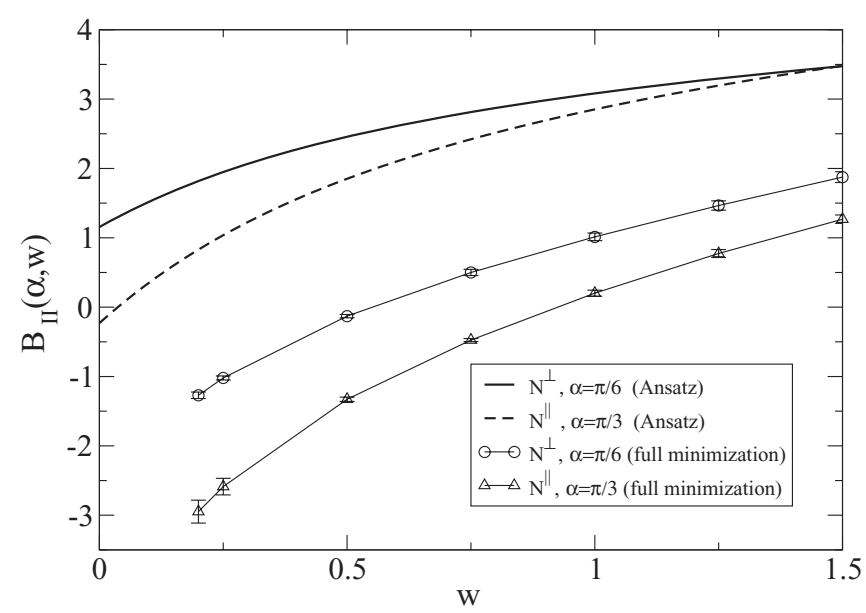

FIG. 9. Comparison between the values of $B_{\text {II }}(\alpha, w)$ obtained from the full minimization of the LdG model and the ansatz (see text). Symbols correspond to $B_{\mathrm{II}}(\alpha, w)$ obtained from the full minimization of the LdG model for $\alpha=\pi / 6$ in the $N^{\perp}$ texture (circles) and $\alpha=\pi / 3$ in the $N^{\|}$texture (up triangles). The wide continuous and dashed lines correspond to the estimates from the ansatz for $\alpha=\pi / 6$ and $\alpha=\pi / 3$, respectively. 
crystals in contact to general substrates [29,30,35]. However, we must note that our analysis is restricted to bulk nematics liquid crystals with in-plane deformations in the presence of grooved substrates. The effect of the substrate structure in the nematic texture in partially filled configurations (i.e., with an isotropic fluid in bulk), the influence of twist nematic deformations [19], and the effect of the structure in the $z$ direction deserve further study, but this is beyond the scope of this work.

\section{ACKNOWLEDGMENTS}

The authors thank P. Patrício and N. M. Silvestre for very stimulating conversations and technical advice on the finiteelement method and M. M. Telo da Gama, L. F. Rull, and A. O. Parry for enlightening discussions. We acknowledge the support from MICINN (Spain) through Grant No. FIS200909326 and Junta de Andalucía (Spain) through Grant No. P09FQM-4938, both cofunded by EU FEDER.

\section{APPENDIX: ANSATZ FOR THE EVALUATION OF $f_{\text {II }}$}

In order to estimate the free energy associated to region II, we can make use of an ansatz. As argued in the paper, the nematic order parameter $S$ must vanish at the cusps of the substrate to avoid the divergence of the free energy, but it must converge to the bulk value far from the wedges and apexes. So, in the region around each cusp, we can suppose that $S$ depends only on the radial distance $r$ from the wedge or apex. In particular, we use the following ansatz [43]: $S(r)=S_{b}\left(1-e^{-\frac{r}{\beta}}\right)$, where $S_{b}$ is the bulk nematic order parameter and $\beta$ is a length scale to be determined later. We will assume that the biaxiality parameter $B=0$ everywhere. Regarding the orientational field $\theta$, we assume that has the expression corresponding to a disclination line placed at the origin, $\theta=\alpha_{\infty}+I(\phi-\pi / 2)$, where $I$ is the winding number associated to the disclination line and $\alpha_{\infty}$ is the far-field value of the orientational field ( 0 for the $N^{\perp}$ texture and $\pi / 2$ for the $N^{\|}$texture). Substituting this ansatz in the $\mathrm{LdG}$ functional [Eq. (5)], this expression reduces to a function of $\beta$,

$$
\begin{aligned}
\lambda f \approx & \frac{13}{144}(\pi \mp 2 \alpha) \beta^{2}-2 w\left(R_{c}-\beta\right)+\frac{1}{7}\left\{9 I^{2}(\pi \mp 2 \alpha)\left[\gamma+\ln \left(\frac{R_{c}}{2 \beta}\right)\right]+\frac{11}{16}(\pi \mp 2 \alpha)\right. \\
& \left.+\frac{1}{8}\left(\frac{\sin [(I-1)(\pi \mp 2 \alpha)]}{(I-1)} \mp 18 I \sin (2 \alpha)-3[\sin (-2 I(\pi \mp \alpha)+(I-1) \pi)-\sin (-2 I( \pm \alpha)+(I-1) \pi)]\right)\right\},
\end{aligned}
$$

where we have considered the case $\tau=1$ and $\kappa=2$ (for other values, we can get straightforwardly analogous expressions). In this expression, the upper sign corresponds to the wedge situation (so $I=I_{1}$ ), and the lower one to the apex (so $I=I_{2}$ ), and $\gamma$ is the Euler constant. Minimizing Eq. (A1) with respect to $\beta$, we obtain the optimal value for this length scale:

$$
\beta=-\frac{72}{13} \frac{w}{(\pi \mp 2 \alpha)}+\sqrt{\left[\frac{72}{13} \frac{w}{(\pi \mp 2 \alpha)}\right]^{2}+\frac{648}{91} I^{2} .}
$$

Substituting Eq. (A2) into Eq. (A1), we get the freeenergy estimate associated to each region around a cusp, which provides an upper limit to the real value of $\lambda f$.

In order to have an estimate of $B_{\mathrm{II}}$, we subtract to the obtained values of $\lambda f$ through this ansatz the surface and elastic contributions $-2 w R_{c}$ and $(K / 2) I^{2}(\pi \mp 2 \alpha) \ln R_{c}=$ $(9 / 7) I^{2}(\pi \mp 2 \alpha) \ln R_{c}$, respectively. After this, the estimate of $B_{\mathrm{II}}$ is obtained as the sum of the results obtained for the wedge and apex. Figure 9 shows the comparison of this estimates with the results obtained from the full minimization for $\alpha=\pi / 6$ ( $N^{\perp}$ texture) and $\alpha=\pi / 3\left(N^{\|}\right.$texture). They have the same order of magnitude, although our ansatz overestimates the value of $B_{\mathrm{II}}$, and, qualitatively, the dependence of $B_{\mathrm{II}}$ with $w$ is recovered.
[1] B.-W. Lee and N. A. Clark, Science 291, 2576 (2001).

[2] J.-H. Kim, M. Yoneya, and H. Yokoyama, Nature 420, 19 (2002).

[3] S. Ferjani, Y. Choi, J. Pendery, R. G. Petschek, and C. Rosenblatt, Phys. Rev. Lett. 104, 257801 (2010).

[4] C. V. Brown, M. J. Towler, V. C. Hui, and G. P. Bryan-Brown, Liq. Cryst. 27, 233 (2000).

[5] C. Uche, S. J. Elston, and L. A. Parry-Jones, J. Phys. D: Appl. Phys. 38, 2283 (2005).

[6] C. Uche, S. J. Elston, and L. A. Parry-Jones, Liq. Cryst. 33, 697 (2006).

[7] A. J. Davidson, C. V. Brown, N. J. Mottram, S. Ladak, and C. R. Evans, Phys. Rev. E 81, 051712 (2010).
[8] C. R. Evans, A. J. Davidson, C. V. Brown, and N. J. Mottram, J. Phys. D: Appl. Phys. 43, 495105 (2010).

[9] O. J. Dammone, I. Zacharoudiou, R. P. A. Dullens, J. M. Yeomans, M. P. Lettinga, and D. G. A. L. Aarts, Phys. Rev. Lett. 109, 108303 (2012).

[10] N. M. Silvestre, P. Patrício and M. M. Telo da Gama, Phys. Rev. E 69, 061402 (2004).

[11] T. Ohzono and J.-i. Fukuda, Nat. Commun. 3, 701 (2012).

[12] D. W. Berreman, Phys. Rev. Lett. 28, 1683 (1972).

[13] P. G. de Gennes and J. Prost, The Physics of Liquid Crystals, 2nd ed. (Oxford University Press, Oxford, 1995).

[14] G. Barbero, Lett. Nuovo Cimento Soc. Ital. Fis. 29, 553 (1980). 
[15] G. Barbero, Lett. Nuovo Cimento Soc. Ital. Fis. 32, 60 (1981).

[16] G. Barbero, Lett. Nuovo Cimento Soc. Ital. Fis. 34, 173 (1982).

[17] S. Kitson and A. Geisow, Appl. Phys. Lett. 80, 3635 (2002).

[18] J. I. Fukuda, M. Yoneya, and H. Yokoyama, Phys. Rev. Lett. 98, 187803 (2007).

[19] P. Patrício, M. M. Telo da Gama, and S. Dietrich, Phys. Rev. Lett. 88, 245502 (2002).

[20] L. Harnau, S. Kondrat, and A. Poniewierski, Phys. Rev. E 72, 011701 (2005).

[21] S. Kondrat, A. Poniewierski, and L. Harnau, Liq. Cryst. 32, 95 (2005).

[22] L. Harnau and S. Dietrich, Europhys. Lett. 73, 28 (2006).

[23] L. Harnau, S. Kondrat, and A. Poniewierski, Phys. Rev. E 76, 051701 (2007).

[24] G. Barbero, A. S. Gliozzi, M. Scalerandi, and L. R. Evangelista, Phys. Rev. E 77, 051703 (2008).

[25] Y. Yi, G. Lombardo, N. Ashby, R. Barberi, J. E. Maclennan, and N. A. Clark, Phys. Rev. E 79, 041701 (2009).

[26] A. Poniewierski, Eur. Phys. J. E 31, 169 (2010).

[27] J. M. Romero-Enrique, C.-T. Pham, and P. Patrício, Phys. Rev. E 82, 011707 (2010).

[28] J. P. Bramble, S. D. Evans, J. R. Henderson, C. Anquetil, D. J. Cleaver, and N. J. Smith, Liq. Cryst. 34, 1059 (2007).

[29] P. Patrício, C.-T. Pham, and J. M. Romero-Enrique, Eur. Phys. J. E 26, 97 (2008).
[30] P. Patrício, J. M. Romero-Enrique, N. M. Silvestre, N. R. Bernardino, and M. M. Telo da Gama, Mol. Phys. 109, 1067 (2011).

[31] P. Patrício, N. M. Silvestre, C.-T. Pham, and J. M. RomeroEnrique, Phys. Rev. E 84, 021701 (2011).

[32] M. Nobili and G. Durand, Phys. Rev. A 46, R6174 (1992).

[33] P. Sheng, Phys. Rev. Lett. 37, 1059 (1976).

[34] F. N. Braun, T. J. Sluckin, and E. Velasco, J. Phys.: Condens. Matter 8, 2741 (1996).

[35] N. M. Silvestre, Z. Eskandari, P. Patrício, J. M. Romero-Enrique, and M. M. Telo da Gama, Phys. Rev. E 86, 011703 (2012).

[36] O. C. Zienkiewicz and R. L. Taylor, The Finite Element Method, 5th ed. (Butterworth-Heineman, Oxford, 2000).

[37] P. Patrício, M. Tasinkevych, and M. M. Telo da Gama, Eur. Phys. J. E 7, 117 (2002).

[38] C. A. Brebbia and J. Domínguez, Boundary Elements: An Introductory Course, 2nd ed. (Computational Mechanics, Southampton, 1992).

[39] J. T. Katsikadelis, Boundary Elements: Theory and Applications (Elsevier, Amsterdam, 2002).

[40] A. J. Davidson and N. J. Mottram, Eur. J. Appl. Math. 23, 99 (2011).

[41] S. Faetti, K. Sakamoto, and K. Usami, Phys. Rev. E 75, 051704 (2007).

[42] P. M. Chaikin and T.C. Lubensky, Principles of Condensed Matter Physics (Cambridge University Press, Cambridge, 1997).

[43] M. Tasinkevych, N. M. Silvestre, P. Patricio, and M. M. Telo da Gama, Eur. Phys. J. E 9, 341 (2002). 